\title{
NOVEL INSIGHTS INTO MANNITOL METABOLISM IN THE FUNGAL PLANT PATHOGEN BOTRYTIS CINEREA
}

Authors: Thierry Dulermo ${ }^{*}$, Christine Rascle ${ }^{*}$, Geneviève Billon-Grand*, Elisabeth Gout ${ }^{\ddagger}$, Richard Bligny ${ }^{\ddagger}$ and Pascale Cotton ${ }^{\S}$

Address

${ }^{*}$ Génomique Fonctionnelle des Champignons Pathogènes des Plantes, UMR 5240 Microbiologie, Adaptation et Pathogénie, Université de Lyon, Lyon, F-69003, France ; Université Lyon1- CNRS-INSA-BayerCropScience, 10 rue Pierre Baizet, F-69009, Lyon, France, †Laboratoire de Microbiologie et Génétique Moléculaire, CNRS UMR 2585, INRA UMR1238, AgroParisTech, INRA, centre de Versailles-Grignon BP 01, F-78850 ThivervalGrignon, France, ¥Physiologie Cellulaire et Végétale, UMR 5168, Institut de Recherche en Technologies et Sciences pour le Vivant, CEA, 17 rue des Martyrs, 38054 Grenoble cedex 9, France, §Génétique Moléculaire des Levures, UMR 5240 Microbiologie, Adaptation et Pathogénie, Université de Lyon, Lyon, F-69003, France ; Université Lyon1- CNRS-INSABayer CropScience, 10 rue Raphaël Dubois, Bât Lwoff, Villeurbanne, F-69621, France

For correspondence. E-mail pascale.cotton@univ-lyon1.fr; Tel +33472448030; Fax +33 472432686

Short title : Mannitol pathway in Botrytis cinerea

\section{SYNOPSIS}

In order to redefine the mannitol pathway in the necrotrophic plant pathogen Botrytis cinerea, we used a targeted deletion strategy of genes encoding two proteins of mannitol metabolism, a mannitol dehydrogenase (BcMTDH), and a mannitol-1-phosphate dehydrogenase (BcMPD). Mobilization of mannitol and quantification of Bcmpd and Bcmtdh gene transcripts during development and osmotic stress confirmed a role for mannitol as temporary and disposable carbon storage compound. In order to study metabolic fluxes, we followed conversion of labelled hexoses by wild type and Bcmpd and Bcmtdh mutant strains by in vivo NMR spectroscopy. Our data revealed that glucose and fructose were metabolized via the BcMPD and BcMTDH pathways, respectively. Existence of a novel mannitol phosphorylation pathway was suggested by NMR investigations. This last finding definitively challenged the existence of the originally postulated mannitol cycle in favor of two simultaneously expressed pathways. Finally, physiological and biochemical studies conducted on double deletion mutants $(B c m p d / B c m t d h)$ showed that mannitol was still produced despite a complete alteration of both mannitol biosynthesis pathways. This strongly suggests that one or several additional undescribed pathways could participate to mannitol metabolism in $B$. cinerea.

Key words:

Botrytis cinerea, in vivo NMR spectroscopy, mannitol, carbon metabolism, osmotic stress, trehalose.

Abbreviations used: BcMPD, Botrytis cinerea MPD; BcMTDH, Botrytis cinerea MTDH; MPD, mannitol-1-phosphate dehydrogenase; MTDH, mannitol dehydrogenase; PCA, perchloric acid; TLC, thin layer chromatography.

\section{INTRODUCTION}


Mannitol is one of the most abundant polyols occurring in nature. It is usually the most abundant soluble carbohydrate within the mycelium [1]. In fungi, several physiological functions have been ascribed to D-mannitol, such as reservoir of reducing power [2], carbon storage compound accumulated in Agaricus bisporus basidiospores [3] or necessary for the formation of fruit bodies in Stagonospora nodorum [4]. Mannitol contributes to stress tolerance in fungi. In Aspergillus niger, mannitol appears to be essential for the protection of spores against cell damage under a variety of stress conditions including cold, drought and oxidative stress [5]. In mycorrhizal fungi, mannitol plays the role of carbon translocation compound that enables fungi to assimilate carbohydrates from plant origin [6, 7]. Furthermore, mannitol has a role in fungal-plant interactions. During the biotrophic interaction Uromyces fabae/Vicia faba, levels of mannitol markedly increase in apoplastic fluids of infected leaves and in spores. Mannitol might have a dual function, sequestration of plant hexoses and protection against reactive oxygen species [8]. A role for mannitol in pathogenicity of Alternaria alternata on its host, tobacco, was demonstrated [9, 10]. Mannitol was secreted by $A$. alternata in response to tobacco extracts. In addition, tobacco expressed an endogenous mannitol dehydrogenase during infection [9, 11]. Mannitol, secreted by A. alternata could play an antioxidant role and quench reactive oxygen species. Mannitol dehydrogenase, induced in plant by fungal colonization, degrades pathogen-produced mannitol, allowing for ROS-mediated plant defense to be effective against the fungus.

The metabolic pathway for mannitol biosynthesis and catabolism (Figure. 1), well described in filamentous fungi, takes place through the mannitol cycle, involving two pathways [12]. The direct reduction of fructose-6-phosphate into mannitol-1-phosphate involves a mannitol-1-phosphate dehydrogenase (MPD, EC 1.1.1.17). Mannitol-1-phosphate is then dephosphorylated into mannitol via a mannitol-1-phosphate phosphatase. This last reaction was described as irreversible, consequently, mannitol degradation is supposed to occur through oxidation of mannitol to fructose via a reversible mannitol dehydrogenase (MTDH, EC 1.1.1.138). Both pathways exist in ascomycetes [2].

However, recent reports have challenged the existence of a mannitol cycle. In S. nodorum, MPD is necessary for mannitol catabolism, whereas MTDH is not [4, 13]. A mannitol phosphorylation pathway allowing conversion of mannitol into mannitol-1-phosphate might exist. Mannitol pathway explorations conducted in S. nodorum and A. alternata revealed that mannitol synthesis occurred mainly through MPD [13, 14]. mpd deletion mutant strains revealed an intracellular mannitol concentration decreased by more than $80 \%$, whereas $m t d h$ strains were almost completely phenotypically wild type. While mannitol metabolism through mannitol-1-phosphate appears to be the dominant route, the physiological role of MTDH branch remains unclear.

In a previous report, we showed that mannitol was accumulated during infection of sunflower by the necrotrophic plant pathogen $B$. cinerea [15]. During pathogenesis, plant hexoses were converted into mannitol, which was the major soluble carbon compound detected in infected tissues. In previous experiments, we were unable to detect mannitol in fungal growth media [15]. This polyol could be used as translocation compound to sequester plant hexoses. Moreover, mannitol accumulated mainly during the final steps of fungal development in planta, being stored when conidiophores emerged. Mannitol could be necessary for spore survival or constitute a source of energy for germination. By means of reverse genetics and metabolic investigations, we have explored the physiological role and functioning of the mannitol pathway in B. cinerea.

\section{EXPERIMENTAL}


100

101

102

103

104

105

106

107

108

109

110

111

112

113

114

115

116

117

118

119

120

121

122

123

124

125

126

127

128

129

130

131

132

133

134

135

136

137

138

139

140

141

142

143

144

145

146

147

148

149

\section{Fungal strain and growth conditions}

Botrytis cinerea B05.10 was maintained at $21^{\circ} \mathrm{C}$ on rich medium as described in [16]. B. cinerea developmental stages were obtained from mycelia grown for 2 or 6 days (mycelium and sporulating mycelium) on cellophane sheets deposited on $2 \%$ glucose-rich solid medium. Conidia were harvested from 12-day-old mycelium grown on $2 \%$ glucose-rich solid medium. Germinating conidia were obtained by cultivating 12-days-old spores $\left(10^{7} \mathrm{ml}^{-}\right.$ ${ }^{1}$ ) in $2 \%$ glucose-rich liquid medium (110 rpm). For osmotic stress experiments, mycelia were grown for 2 days on cellophane on solid Gamborg medium ( $\mathrm{pH} 5.0$ ) supplemented with $2 \%$ glucose [16]. Mycelia were then transferred for $30 \mathrm{~min}, 1 \mathrm{~h}, 4 \mathrm{~h}, 9 \mathrm{~h}$, and $24 \mathrm{~h}$ on fresh solid medium supplemented with $2 \%$ glucose and $1 \mathrm{M} \mathrm{NaCl}$. For osmotic stress response analysis by in vitro NMR spectroscopy, mycelia were first transferred for $4 \mathrm{~h}$ on labelled fructose plates $\left(1 \%\right.$ fructose containing $10 \%{ }^{13} \mathrm{C}$-fructose) and transferred for 1 and $4 \mathrm{~h}$ on fresh solid medium supplemented with $2 \%$ glucose and $1 \mathrm{M} \mathrm{NaCl}$. Mycelia, conidia and germinating conidia were harvested, frozen in liquid nitrogen then stored at $-80^{\circ} \mathrm{C}$. In vivo NMR analyses were performed in a perfusion medium containing $1 \mathrm{~g} \mathrm{l}^{-1} \mathrm{KNO}_{3}, 0.05 \mathrm{~g} \mathrm{l}^{-1} \mathrm{KCl}, 0.1 \mathrm{~g} \mathrm{l}^{-1}$ $\mathrm{MgSO}_{4}, 0.1 \mathrm{~g} \mathrm{l}^{-1} \mathrm{CaSO}_{4}$, and ${ }^{13} \mathrm{C}$-labelled glucose or fructose as indicated in text. B. cinerea transformant strains were selected on rich media supplemented with $70 \mu \mathrm{g} \mathrm{ml}^{-1}$ hygromycin (Invivogen, Toulouse, France) or with $100 \mu \mathrm{g} \mathrm{ml}^{-1}$ nourseothricin (Werner BioAgents, Jena, Germany).

\section{In vitro germination and conidiation assays}

Conidia were harvested in $0.5 \%$ Tween 80 from 12-days-old mycelium grown on solid rich medium. For in vitro germination assays, 500 conidia of each strain were spread on solid rich medium in $140 \mathrm{~mm}$ plates. Young germinations were counted after 4 days of incubation. For conidiation assays, 1000 conidia were spread on solid rich medium in 24-well-plates. After 12 days of growth, conidia produced in each well were harvested and counted.

\section{Pathogenicity tests}

Phytopathogenicity assays were performed on sunflower cotyledons as hosts as previously described [15]. Cotyledons from 1-week-old germlings were infected at the end of a dark period by depositing a 5-mm-mycelium disk near the tip of the leaves. Necrosis was detectable 24 hpi (hours post infection) by the appearance of a brown color surrounding the starting point of infection. At $48 \mathrm{hpi}$, half of the cotyledons were macerated and necrosed. The whole cotyledon was infected at 72 hpi. Conidiation began at 96 hpi and was achieved at 120 hpi.

\section{Plasmid construction and transformation of $B$. cinerea}

Transformation of $B$. cinerea $\mathrm{B} 05.10$ was performed using the $h p h$ gene as selectable marker. To disrupt Bcmpd and Bcmtdh, constructs containing the hygromycin resistance cassette (OliC promoter- $h p h$ gene coding sequence- tublterminator) flanked by 5' and 3'Bcmpd and $B c m t d h$ genomic DNA fragments were realized (Figure S1). The hygromycin cassette was released from pLOB1 (provided by P. Tudzynski, Münster, Germany) after EcoRI and EcoRV digestion, and then cloned in pBKSII. Flanking regions (5'Bcmpd, 3 'Bcmpd, 5 'Bcmtdh and 3 'Bcmtdh fragments) amplified using $\mathrm{P} 1 / \mathrm{P} 2, \mathrm{P} 3 / \mathrm{P} 4, \mathrm{P} 5 / \mathrm{P} 6$ and $\mathrm{P} 7 / \mathrm{P} 8$ primers respectively (Table S1 and Figure S1) were cloned on every side of the hygromycin cassette (Figure S1) to form pTD5 and pTD6. To obtain pTD10 plasmid, Bcmtdh 5' and 3' flanking regions were 
150 amplified using P9/P10 and P11/P12 respectively (Figure S1). Amplified fragments were 151 cloned in pCB04, on each side of the nourseothricin cassette, which contained the 152 Streptomyces nourseothricin N-acetyl-transferase gene (nat1) flanked by the $A$. nidulans OliC 153 promoter and the $B$. cinerea tubl terminator [17]. Preparation of protoplasts and 154 transformation were adapted from procedures described in [18]. Fungal cell wall was digested using $50 \mathrm{mg} \mathrm{ml}^{-1}$ Glucanex (Novozymes, Dittengen, Switzerland) for $2 \mathrm{~h}$ at $26^{\circ} \mathrm{C}$. Wild type strain (WT) was transformed with $10 \mu \mathrm{g}$ of Not linearized vector pTD5 or pTD6 to obtain Bcmtdh and Bcmpd mutants respectively. The development of a double mutant was achieved by transforming the previously generated $\triangle B c m p d$ strain with $10 \mu \mathrm{g}$ of $S c a$ linearized vector pTD10. PCR analyses were performed to ensure replacement of gene of interest by the selection cassette. gDNA from Bcmpd transformants was amplified using P13/P14 and P15/P16 primers (Table S1), to check 5' and 3' insertions respectively. gDNA from Bcmtdh transformants was amplified using P17/P14 primers and P18/P19 primers to verify 5' and 3' insertions respectively (Table S1). Bcmtdh gene replacement by the nourseothricin cassette was verified using primers $\mathrm{P} 20 / \mathrm{P} 21$ and $\mathrm{P} 22 / \mathrm{P} 19$ to check $5^{\prime}$ and $3^{\prime}$ insertions respectively (Table S1). Southern analyses were performed to ensure single insertions. DNA (5 $\mu \mathrm{g})$ was digested with EcoRI for single mutants, and with HindIII for double mutants, separated through an agarose gel $(0.8 \%)$ and transferred to a nylon membrane. A ${ }^{32} \mathrm{P}$-labelled probe, ( $h p h$ coding sequence for single mutants or the 5'Bcmtdh flanking region for double mutants), was obtained from plasmids pLOB1 and pTD5 using Megaprime DNA Labelling system (Amersham Biosciences). Hybridization was carried out as described in [19].

\section{RNA isolation and transcript quantification}

Biological materials were frozen in liquid nitrogen and kept at $-80^{\circ} \mathrm{C}$. Samples were crushed in liquid nitrogen, and total RNAs were extracted by phenol/chloroform separation and lithium chloride precipitation [20]. For Q-PCR experiments, $20 \mu \mathrm{g}$ of total RNA of each sample were treated with DNAseI (Ambion). Absence of genomic DNA was controlled by PCR using the DNaseI-treated total RNA as template and Taq-DNA polymerase (MP Biomedicals, Solon, USA). Quality of total RNA was verified using Agilent 2100 Bioanalyzer, Agilent RNA 6000 Nano reagents and RNA Chips. Total DNaseI-treated RNA $(5 \mu \mathrm{g})$ was treated with Thermoscript RT (Invitrogen, Carlsbad, USA) as described by manufacturer. Q-PCR experiments were performed in ABI PRISM 7900HT (Applied Biosystems, Foster City, USA) using the Power SYBR ${ }^{\circledR}$ Green PCR Master Mix (Applied Biosystems) according to the instructions of the manufacturer. Relative quantification was based on the $2^{\Delta \mathrm{CT}}$ method using Bcact1 and BctubA as calibrator references. As gene expression profiles were similar using both controls, only the results obtained using BcactA transcripts are presented here. The amplification reaction was as follows: $95^{\circ} \mathrm{C} 10 \mathrm{~min}, 95^{\circ} \mathrm{C}$ $15 \mathrm{~s}$, and $60^{\circ} \mathrm{C} 1 \mathrm{~min}(50 \mathrm{cycles}), 95^{\circ} \mathrm{C} 15 \mathrm{~s}, 60^{\circ} \mathrm{C} 15 \mathrm{~s}$, and $95^{\circ} \mathrm{C} 15 \mathrm{~s}$. Three independent replicates, prepared from independent biological samples, were analyzed. Primers used for QPCR are shown in Table S1.

\section{NMR spectroscopy}

PCA (perchloric acid) extracts were prepared from 5 to $10 \mathrm{~g} B$. cinerea mycelia according to the method described in [21]. Values are given in $\mathrm{mg} \mathrm{g}^{-1}$ fresh weight (FW) of fungal material. In vitro (tissue extracts) and in vivo (perfused tissues) spectra were recorded on a Bruker NMR spectrometer (AMX 400, wide bore; Bruker, Billerica, MA) equipped either with a $10-\mathrm{mm}$ or a $25-\mathrm{mm}$ multinuclear-probe tuned at 161.9 or $100.6 \mathrm{MHz}$ for ${ }^{31} \mathrm{P}$ - and ${ }^{13} \mathrm{C}$ analyses. The deuterium resonance of ${ }^{2} \mathrm{H}_{2} \mathrm{O}$ was used as lock signal. For in vitro 
200 measurements, ${ }^{13} \mathrm{C}-\mathrm{NMR}$ and ${ }^{31} \mathrm{P}-\mathrm{NMR}$ acquisitions were performed as described in [15]. 201 Assignments and quantifications were made after running a series of spectra of extracts, 202 added with known amounts of authentic compounds, and at differents pHs for the extracts 203 submitted to ${ }^{31} \mathrm{P}-\mathrm{NMR}$ analysis, in particular to be certain that the peak of resonance 204 appearing at $4.77 \mathrm{ppm}$ in samples incubated with mannitol corresponded well to mannitol-1205 phosphate. In vivo measurements were performed using $48 \mathrm{hpi}$ sunflower cotyledons as 206

207

208

209

210

\section{Polyol and sugar detection by thin layer chromatography (TLC)}

Mannitol was extracted from conidia, germinating conidia, mycelia or conidiating mycelia frozen in liquid nitrogen, crushed and then lyophilized. In order to obtain comparable data, $100 \mathrm{mg}$ of dessicated fungal extracts were suspended in $900 \mu \mathrm{l}$ of water and boiled for $10 \mathrm{~min}$ for each sample. Supernatants were analyzed by thin layer chromatography (TLC). One $\mu 1$ of each sample was deposited on TLC-plates SIL G-25 (Macherey-Nagel, France) and separated in acetonitril:ethylacetate:propanol:water (85:20:20:15, by vol). Sugars and polyols were revealed using $0,5 \% \mathrm{KMnO}_{4}, 1 \mathrm{~N} \mathrm{NaOH}$ and identified by using standard sugars or polyols (1 $\mu \mathrm{l}$ of $0.5 \%$ standard solution).

\section{Preparation of cell extracts and enzyme assays}

Cell extracts were prepared as described in [5]. Lyophilized mycelia were ground, resuspended in extraction buffer (50 mM phosphate buffer $\mathrm{pH} 7.0,0.5 \mathrm{mM}$ EDTA, 5mM $\beta-$ mercaptoethanol), then centrifuged for $30 \mathrm{~min}$ at $15000 \mathrm{rpm}$ at $4{ }^{\circ} \mathrm{C}$. Supernatants were collected and desalted on a Zeba Desalt Spin column (ThermoScientific, Cergy Pontoise, France) before enzyme activity measurements. Enzyme assays were performed as described in [22] using $50 \mu \mathrm{g}$ of proteins in a final volume of $1 \mathrm{ml}$ and by monitoring absorbance changes of $\mathrm{NAD}(\mathrm{P})+/ \mathrm{NAD}(\mathrm{P}) \mathrm{H}$ at $340 \mathrm{~nm}$. For $\mathrm{MTDH}$ activity, the reduction of fructose to mannitol was assayed in a reaction mixture containing $0.6 \mathrm{M}$ fructose, $0.2 \mathrm{mM}$ NADPH, 10 $\mathrm{mM}$ HEPES $\mathrm{pH}$ 7.0. The oxidation of mannitol to fructose was conducted using $0.4 \mathrm{M}$ mannitol, 2mM NADP, 10 mM HEPES pH 9.0. For MPD activity, the reduction of fructose6-phosphate was assayed in a reaction mixture containing $5 \mathrm{mM}$ fructose-6-phosphate, 0.3 $\mathrm{mM}$ NADH, $10 \mathrm{mM}$ Hepes $\mathrm{pH}$ 7.0. For the reverse reaction, the oxidation of mannitol-1phosphate was measured using $5 \mathrm{mM}$ mannitol-1-phosphate, $0.5 \mathrm{mM}$ NAD, $10 \mathrm{mM}$ HEPES $\mathrm{pH} 9.0$. Enzyme activity was expressed as nmoles of cofactor $(\mathrm{NAD}(\mathrm{P}) \mathrm{H}$ reduced or oxidized) per minute and per $\mu \mathrm{g}$ of total cell extract protein.

\section{Western Blot analysis}

BcMPD and BcMTDH were produced as His-tag fusion proteins in E.coli M15 using the QiaExpress System (Qiagen, Courtaboeuf, France). cDNAs were amplified by PCR with forward primers containing $N c o$ I restriction sites and reverse primers containing BglII sites (Table S1). PCR products were cloned in pQE60. Optimal protein expression was achieved using $2 \mathrm{mM}$ IPTG after $4 \mathrm{~h}$ of induction at $37^{\circ} \mathrm{C}$ and $1 \mathrm{mM}$ IPTG after $20 \mathrm{~h}$ of induction at $23^{\circ} \mathrm{C}$, for BcMPD and BcMTDH respectively. BcMPD was detected in inclusion bodies while $\mathrm{BcMTDH}$ remained in soluble fraction. Purification of tagged proteins was performed on NiNTA columns, under denaturing and native conditions for BcMPD and BcMTDH respectively, according to manufacturer's instructions. Anti-BcMPD and anti-MTDH sera were obtained by immunization of rabbits with the purified proteins (Covalab, Villeurbanne, France). Detection of BcMPD and BcMTDH in fungal strains was performed by Western blot 
250

251

252

253

254

255

256

257

258

259

260

261

262

263

264

265

266

267

268

269

270

271

272

273

274

275

276

277

278

279

280

281

282

283

284

285

286

287

288

289

290

291

292

293

294

295

296

297

298

299

analyses. Total cell extract proteins were quantified in order to load $75 \mu \mathrm{g}$ of each extract in each lane. Proteins were then separated by SDS-PAGE in a $10 \%$ acrylamide gel and blotted onto a nitrocellulose membrane. Nitrocellulose membranes were probed with rabbit polyclonal BcMPD (1:2500) and BcMTDH (1:2500) antisera. Bands were visualized with ECL (ElectroChemiLuminescence) using HRP (horseradish peroxidase) conjugated with goat anti-rabbit IgG (dilution 1:40000). The detection was performed as described in the manufacturer's instructions for the ECL Western detection kit (Super Signal West Pico Chemiluminescent Substrate, Perbio, France).

\section{RESULTS AND DISCUSSION}

\section{Construction of Bcmpd and Bcmtdh mutant strains}

To understand the role of mannitol in B. cinerea (Figure 1), we searched for mannitol dehydrogenase and mannitol-1-phosphate dehydrogenase coding sequences, in order to construct single and double deletion mutant strains. Bcmtdh and Bcmpd genes were identified in the genome sequence of B. cinerea (http://www.broad.mit.edu/annotation/genome/botrytis_cinerea/). Low stringency Southern blot indicated that only one copy of each gene was present in the $B$. cinerea genome (data not shown). Each sequence (respectively $1314 \mathrm{bp}$ and $862 \mathrm{bp}$ for Bcmpd and Bcmtdh) had one intron. Sequence analyses revealed that BcMPD and BcMTDH had the features of mannitol1-phosphate 5-dehydrogenases and mannitol 2-dehydrogenases and coded respectively 43 and $28 \mathrm{kDa}$ isoforms. BcMDTH shares $71 \%$ and $78 \%$ identity with S. nodorum Mdh1 and A. alternata $\mathrm{MtDH}$, respectively. BcMPD shares $62 \%$ and $59 \%$ identity with S. nodorum Mpd1 and A. alternata MpDH, respectively. Whereas BcMPD belongs to the long-chain dehydrogenases/reductases superfamily (LDR), BcMTDH falls into the short-chain group of dehydrogenase/reductase superfamily [23-24].

In the Bcmpd deletion construct, a region including $567 \mathrm{bp}$ of the promoter, the open reading frame (ORF) and 328 bp of the $3^{\prime}$ of the gene was replaced by a hygromycin resistance cassette. In the case of the Bcmtdh deletion vector, $60 \mathrm{bp}$ of the promoter and $423 \mathrm{bp}$ of the coding sequence were replaced by the hygromycin resistance cassette (Figure S1A, B). Protoplasts of B. cinerea B05.10 strain were transformed with the linearized vectors and hygromycin-resistant transformants were tested by PCR and Southern analyses (data not shown, Figure S1D). In both cases, two transformants, $\Delta B c m p d 19$ and $\Delta B c m p d 24$, for $B c m p d$, and $\triangle B c m t d h 11$ and $\triangle B c m t d h 16$, for $B c m t d h$, were found to have undergone gene replacement as expected (Figure S1D). Gene deletions were further confirmed through transcriptional analyses and western blot that revealed an absence of transcripts and proteins in the mutants as compared to the wild type strain (Figure 2A, B). Interestingly, as compared to the wild type strain, Bcmtdh expression was clearly increased in Bcmpd mutant, whereas $B c m p d$ transcription level was not affected by Bcmtdh deletion. Western blot experiments showed that BcMPD and BcMTDH proteins seemed to be more abundant in both deletion mutants (Figure 2B). Enzymatic assays (Figure 2C) confirmed that Bcmpd deletion abolished mannitol-1-phosphate dehydrogenase activity. However, in the Bcmtdh mutant, mannitol dehydrogenase activity was strongly reduced but not completely eliminated (Figure 2C). Indeed, a low mannitol dehydrogenase activity remained in Bcmtdh mutant. All experiments presented in this work were conducted on Bcmpd19 and Bcmpd24 for BcMPD pathway and 
300

301

302

303

304

305

306

307

308

309

310

311

312

313

314

315

316

317

318

319

320

321

322

323

324

325

326

327

328

329

330

331

332

333

334

335

336

337

338

339

340

341

342

343

344

345

346

347

348

349

on Bcmtdh11 and Bcmtdh16 for BcMTDH. In both cases, results were clearly similar, in all experiments, for the two mutants analyzed. Consequently, in order to improve reading of the manuscript, results presented here concern Bcmpd19, Bcmtdh16 deletion mutant strains, only.

\section{Conversion of hexoses by BcMPD and BcMTDH pathways}

To investigate the respective role of BcMPD and BcMTDH branches in mannitol metabolism, hexose assimilation and conversion through the mannitol pathway were performed in a plant infection context, by in vivo NMR spectroscopy. Transfer rates of ${ }^{13} \mathrm{C}_{1}$-glucose or ${ }^{13} \mathrm{C}_{2^{-}}$ fructose by $\triangle B c m p d$ or $\triangle B c m t d h$ strains were followed in real time using sunflower cotyledons infected by $B$. cinerea (Table 1 ) and compared to the data previously obtained with the wild type strain [15]. ${ }^{13} \mathrm{C}_{1}$-glucose was converted by $\triangle B c m p d$ and $\triangle B c m t d h$ mutant strains into ${ }^{13} \mathrm{C}_{1}$-trehalose, glycogen and ${ }^{13} \mathrm{C}_{1 / 6}$-mannitol as previously shown [15]. Whereas transfer of ${ }^{13} \mathrm{C}_{1}$ from glucose to mannitol was strongly impaired in $\triangle B c m p d$, amounts of labelled trehalose and glycogen were increased. In $\Delta B c m t d h$, labelling transfer rate of ${ }^{13} \mathrm{C}_{1}$ from glucose into ${ }^{13} \mathrm{C}_{1 / 6}$ mannitol was similar to the WT (wild type) strain, while the amounts of labelled trehalose and glycogen were seriously lowered. ${ }^{13} \mathrm{C}_{2}$-fructose was converted by all the strains in a unique compound, mannitol. However, $\Delta B c m t d h$ transferred ${ }^{13} \mathrm{C}_{2}$ from fructose to mannitol with a lower efficiency, as compared to the WT strain ( $30 \%$ decrease). In vivo NMR data showed trehalose (and glycogen) labelling during growth in the presence of glucose but not in the presence of fructose. Our data clearly demonstrate that trehalose accumulation is a consequence of MPD pathway deletion, preferentially used during glucose assimilation by $B$. cinerea. Trehalose (and glycogen) synthesis is directly linked to glucose-6phosphate (Figure 1). Mannitol biosynthesis is also connected to this metabolite via fructose6-phosphate, used as substrate by BcMPD. Consequently, mannitol synthesis from glucose by BcMPD pathway could deplete the pool of glucose-6-phosphate. Bcmpd mutants, affected in mannitol biosynthesis, could produce trehalose and glycogen to prevent glucose-6-phosphate accumulation.

\section{Mannitol is mobilized during in vitro development of $B$. cinerea}

Metabolic profiling revealed that mannitol is found in large amounts in developing $B$. cinerea mycelium [15]. To try to determine the role of mannitol in $B$. cinerea development, we analyzed sporulation and germination rate. No obvious differences were observed between the WT and single mutant strains (Figure S2). This lack of effect of mannitol gene deletion on sporulation and/or spore germination, prompted us to analyze sugar and polyol content by TLC (thin layer chromatography), after growth in the presence of glucose (Figure 3). For this purpose, fungal extracts were analyzed during distinctive phases of development: growing mycelium (2-day-old cultures), conidiation (6-day-old cultures), mature conidia (collected from 12-day-old cultures) and germination of conidia (performed for 2, 4 and 6 hours).

Mannitol, and to a lesser extent trehalose, were accumulated during sporulation and in mature conidia in WT strain. These compounds, rapidly degraded during germination, were almost undetectable 2 hours after germination initiation (Figure $3 \mathrm{~A}$ ). While $\triangle B c m t d h$ exhibited a similar profile to WT, sugar profile revealed by TLC was modified in $\triangle B c m p d$. Mannitol content was markedly reduced in mycelium, whereas trehalose store drastically increased in mature conidia and mycelium (2-d and 6-d old). Surprisingly, TLC profiles revealed an almost unaltered mannitol content in mutant strain conidia.

Bcmpd and Bcmtdh gene expression was also checked during development phases, in the WT strain. The results presented in Figure $3 \mathrm{~B}$ show that both genes exhibited similar 
expression patterns during in vitro development of WT strain. Despite the dissimilar expression levels, their transcription was particularly high during spore genesis (6-day-old cultures) and in mature conidia. In contrast, during germination, transcript and protein levels drastically decreased (Figure 3B, C). It should be noticed that BcMTDH detection was higher, particularly two hours after germination initiation. This could be explained by a major role played by this protein in mannitol degradation during germination. Nevertheless, both mannitol metabolism branches could participate in mannitol accumulation in conidiating mycelium and spores.

Given its high abundance in spores, the idea of mannitol acting as carbohydrate reserve, as suggested in several reports, can be considered $[25,26]$. Data on sporulation and germination rate in fungal pathogens affected in mannitol metabolism by gene disruption strategies are controversial. In $S$. nodorum, $m p d$ and $m p d / m t d h$ mutants, the production of conidia was strongly affected $[4,13]$, whereas in A. alternata, $m p d / m t d h$ mutant is affected only[10]. As $S$. nodorum and A. alternata, B. cinerea accumulates mannitol in sporulating mycelium $[4,10]$ but, despite a lower mannitol content, mutants were not affected for in vitro or in planta conidiation (Figure S2). A reduced mannitol concentration could allow sporulation. Moreover, trehalose could be stored or degraded instead of mannitol.

\section{Mannitol is involved in osmotic stress response in $B$. cinerea}

To further dissect the mannitol pathway, it was necessary to identify physiological conditions that could induce synthesis or degradation of this compound in $B$. cinerea. An osmotic shock is known to induce remobilization of intracellular carbon pool in fungi [27]. Most reports have so far excluded a role for mannitol in enabling fungi to cope with osmotic stress. Sclerotinia sclerotiorum, Saccharomyces cerevisiae and A. nidulans store glycerol in response to a hyperosmotic stress [28, 29, 30], whereas Magnaporthe grisea, Cladosporium fulvum and $S$. nodorum accumulate arabitol $[31,32,33]$. In $B$. cinerea, osmotic stress response is controlled through glycerol accumulation [34]. But mannitol, directly connected to central carbon metabolism could also be implicated in osmotic stress response. To address this question, we investigated the polyol content of B. cinerea WT, Bcmpd and Bcmtdh mutant strains during growth under hyperosmotic conditions (Figure 4A).

In WT strain, mannitol was transiently degraded during stress response. Its concentration decreased between 1 and $4 \mathrm{~h}$ of stress and then increased from 9 to $24 \mathrm{~h}$ after transfer. In parallel, glycerol accumulated as mannitol was degraded (Figure 4A). Mannitol degradation during osmotic stress response could suggest its contribution to osmo-adaptation. Analysis of Bcmpd and Bcmtdh gene expression by Q-PCR, revealed that Bcmpd was transiently downregulated, whereas $B c m t d h$ transcription increased as mannitol pool decreased (Figure 4B). After one hour of stress, Bcmtdh gene expression reached its maximal level. A 4.5 fold expression increase was detected. BcMPD and BcMTDH protein profiles were analyzed by Western blot (Figure 4A). During osmotic stress, BcMPD was degraded from 0 to $1 \mathrm{~h}$ whereas BcMTDH was accumulated. Hence, BcMTDH could be preferentially dedicated to mannitol degradation during osmotic stress response.

In $\triangle B c m t d h$ mutant, TLC profile was similar to that of the WT strain (Figure 4A). $\triangle B c m p d$ showed a constant and low mannitol content, whereas trehalose, produced by this mutant strain was degraded concomitantly to glycerol accumulation. While in the WT and in the $B c m t d h$ mutant, mannitol pool was partially restored from 9 to $24 \mathrm{~h}$ of stress, trehalose pool was not restored in $\triangle B c m p d$ mutant. Expression profiles of Bcmpd or Bcmtdh genes in WT and both mutant strains were similar (Figure 4B). However, western blot experiments showed that, in $\triangle B c m t d h$ strain, BcMPD was not degraded during osmotic stress (from 0 to $4 \mathrm{~h}$ ). Therefore, while transcriptional control of $B c m p d$ gene was the same, post-translational 
400

401

402

403

404

405

406

407

408

409

410

411

412

413

414

415

416

417

418

419

420

421

422

423

424

425

426

427

428

429

430

431

432

433

434

435

436

437

438

439

440

441

442

443

444

445

446

447

448

449

control differed in WT and $\Delta B c m t d h$ mutant. Mannitol content decreased during osmotic stress response in $\Delta B c m t d h$. This could be due to the low MTDH activity still detected in $\triangle B c m t d h$ cell extracts. On the other hand, BcMPD could participate in mannitol degradation. Consequently, this could suggest, contrary to the proposed cycle [12], that the mannitol-1phosphate dephosphorylation reaction could be reversible to allow mannitol degradation through MPD pathway (Figure 1).

Our data showed that mannitol degradation in WT and Bcmtdh strains paralleled glycerol accumulation during osmotic stress response, suggesting that mannitol could constitute a carbon store in which $B$. cinerea could draw, in order to quickly synthesize glycerol for osmoprotection. For this purpose, mannitol pool of $B$. cinerea wild type strain was labelled using ${ }^{13} \mathrm{C}_{2}$-fructose (fructose is almost solely converted in mannitol in WT strain). An osmotic shock $(1 \mathrm{M} \mathrm{NaCl})$, in the presence of unlabelled glucose, was applied to mycelium and allocation of labelled carbon during osmotic response was then followed by in vitro NMR spectroscopy (Figure S3). Before osmotic stress, labelled carbon was found exclusively in mannitol molecules $\left(\mathrm{C}_{2}\right.$ and $\left.\mathrm{C}_{5}\right)$. Between 1 and $4 \mathrm{~h}$ of stress, mannitol was degraded and $50 \%$ of labelled carbon originating from mannitol was allocated for glycerol synthesis and detected in ${ }^{13} \mathrm{C}_{2}$ glycerol. This result demonstrates that mannitol is not directly implicated in osmotic stress response but could contribute indirectly to osmoprotection via carbon relocation to glycerol molecules. This direct connection between mannitol and glycerol for stress answer underlines the central role of mannitol in B. cinerea carbon metabolism. In WT and single mutants, mannitol and trehalose disappearance correlated to glycerol accumulation (Figure 4). In order to participate to this transient response, trehalose could also be mobilized into glycerol.

\section{Bcmpd deletion mutant reveals a mannitol phosphorylation pathway}

Radial growth experiments revealed that Bcmtdh mutants were able to grow as well as the WT strain on mannitol, used as sole carbon source (Figure S4). Moreover, mannitol degradation occurred in $\triangle B c m t d h$ during osmotic stress response and during germination of spores. This degradation could be performed by the remaining MTDH activity detected in $\Delta B c m t d h$ or reveal that another pathway to degrade mannitol, via mannitol-1-phosphate probably, may exist. To confirm this hypothesis, WT, $\triangle B c m p d$ and $\triangle B c m t d h$ were grown for two days in the presence of $2 \%$ mannitol. Metabolic spectra of mycelial PCA extracts were then analyzed by in vitro ${ }^{31} \mathrm{P}-\mathrm{NMR}$ spectroscopy. Mannitol-1-phosphate peaks were detected at $4.77 \mathrm{ppm}$ for each strain. ${ }^{31} \mathrm{P}$ spectra of $\triangle B c m p d$ are presented in Figure 5. Mannitol-1-phosphate was detected after growth on mannitol, only. MPD is the only known enzyme in fungi able to produce mannitol-1-phosphate from fructose-6-phosphate [1]. Consequently, biosynthesis of mannitol-1-phosphate by $\triangle B c m p d$ requires a pathway which could phosphorylate mannitol into mannitol-1-phosphate. Moreover, Bcmpd and Bcmtdh gene expression analysis, performed after growth of WT strain on synthetic medium containing $2 \%$ mannitol as sole carbon source confirmed this hypothesis. In this case, Bcmpd expression level was 35 fold higher than Bcmtdh (Figure S5). This suggests that mannitol catabolism could occur through phosphorylation of mannitol and subsequent conversion in fructose-6-phosphate via MPD pathway.

Mannitol metabolism in fungi was proposed to occur as a cycle [12]. In this cycle, mannitol is synthesized through MPD pathway and degraded via MTDH pathway. However, several recent reports do not support the metabolism of mannitol operating as a cycle $[4,14]$. The ability of the $\triangle B c m p d$ strains to use mannitol as sole carbon source clearly indicates that mannitol can be metabolized through other routes, most probably through mannitol-1- 
450

451

452

453

454

455

456

457

458

459

460

461

462

463

464

465

466

467

468

469

470

471

472

473

474

475

476

477

478

479

480

481

482

483

484

485

486

487

488

489

490

491

492

493

494

495

496

497

498

499

phosphate. The remaining objection was the absence of mannitol kinase activity in fungi [2, $25]$. In the present work, detection of mannitol-1-phosphate when $\triangle B c m p d$ mutants were grown on mannitol reveals for the first time the occurrence of a mannitol phosphorylation pathway in fungi. As a matter of fact, this phosphorylation of mannitol seems specific to fungi (at least to $B$. cinerea), since it has not been detected in any vascular plant cells or tissues that were preincubated in the presence of mannitol (Bligny, unpublished data). Accumulation of mannitol-1-phosphate could result from a one step reaction involving a mannitol kinase and ATP, or from a series of several enzymatic reactions that remain to be discovered.

\section{Deletion of BcMPD and BcMTDH pathways did not abolish mannitol metabolism in $B$. cinerea and reveal a new metabolic route}

Development and pathogenicity were not affected in $\Delta B c m p d$ and $\Delta B c m t d h$ mutant strains (Figure S2). In vitro and in planta conidiation and in vitro spore germination tests showed that mutant strains were not affected in those developmental steps. In that case, deletion of whole pathway by creating a Bcmpd/Bcmtdh double mutant was indispensable to assign a physiological role to mannitol.

To construct such a double deletion strain, Bcmpd mutant strain was transformed with a Bcmtdh disruption cassette containing a nourseothricin resistance marker (Figure S1A, B, C). To check deletion and homologous recombination, nourseothricin-resistant strains were screened by PCR (data not shown) and Southern blot (Figure S1D). Two strains, $d b 32.3$ and $d b 35.2$ were selected as double gene replacement mutants. Transcriptional analyses and western blotting confirmed deletion of both genes in the double mutants (Figure 2A, B). However, as found in the $\Delta B c m t d h$ single mutant, mannitol dehydrogenase activity was still detected in the double mutant strains, with a reduction of $70 \%$, as compared to the wild type strain (Figure 2C).

Radial growth experiments revealed that the double mutants were able to grow as well as the WT strain on mannitol as sole carbon source (Figure S4). Furthermore, germination and sporulation and infection process were not altered in $\Delta B c m p d \Delta B c m t d h$ as compared to the WT strain (Figure S2). TLC profiles of intracellular sugars and polyols performed during development of $\triangle B c m p d \triangle B c m t d h$ revealed that mannitol was still produced in double mutants (Figure 6). While mannitol store was severely lowered in mycelium it was not affected in conidia (Figure 6). Quantification of intracellular metabolites by in vitro NMR spectroscopy in 2-day-old $\triangle B c m p d \triangle B c m t d h$ mycelium revealed that mannitol was synthetized from glucose and fructose (Table 2). As compared to the WT strain, mannitol content in $\triangle B c m p d \Delta B c m t d h$ mycelium after growth on glucose or fructose was decreased by $70 \%$ and $31 \%$, respectively. The low mannitol dehydrogenase activity still detected in double mutant strains could explain why these mutants are still able to produce mannitol, principally from fructose. Moreover, this compound was still degraded during double mutant spore germination (Figure 6). This may suggest that the remaining mannitol dehydrogenase pathway could compensate mannitol production in double mutant conidia rather than in mycelium and be implicated in mannitol degradation during germination. Theses last findings strongly suggest that mannitol could be synthesized and degraded through potentially other metabolic routes.

Our data are in agreement with those reported in the case of A. alternata $\mathrm{mtdh} / \mathrm{mpd}$ double mutant that was still able to grow on mannitol as sole carbon source [14]. In that case, the authors suggested that the fungus contain other enzymes that allow utilization of mannitol as a substrate. The ability of $B$. cinerea double mutant to metabolize mannitol, together with the presence of a mannitol dehydrogenase activity, suggest that additional unrelated mannitol 
500 dehydrogenase encoding gene(s) should be present in $B$. cinerea genome. Recently, a new 501 mannitol dehydrogenase showing no similarity with any known fungal MTDH has been 502 described in Tuber borchii [35]. The phylogenetic analysis showed TbMDH to be the first 503 example of a fungal mannitol dehydrogenase belonging to the medium-chain 504 dehydrogenases/reductases (MDRs). Consequently, the T. borchii enzyme identified a new 505 group of proteins forming a distinct subfamily of polyol dehydrogenase [35]. A BlastP search 506 (http://www.broadinstitute.org/annotation/genome/botrytis_cinerea/Home.html) for possible 507 homologies with T. borchii Tbmdh mannitol dehydrogenase [35] was thus performed. 508 Surprisingly, our BlastP results clearly pointed out a $B$. cinerea sequence (BC1G_15343), 509 annotated as an alcohol dehydrogenase, sharing $84 \%$ identity ( $89 \%$ positive) with TbMDH, 510 while it shared only $11 \%$ identity with BcMTDH. In T. borchii, TbMDH produces mannitol from fructose. Such an enzyme, with a high preference for fructose and mannitol is a good candidate to perform mannitol synthesis from fructose and degrade mannitol during development in double mutant $B$. cinerea strains. However, further experiments are necessary to clearly implicate this new gene in $B$. cinerea mannitol metabolism.

In conclusion, a physiological role has been assigned to MTDH pathway that could be dedicated to a favourable conversion of fructose to mannitol and to mannitol degradation during osmotic stress response. On the other hand, we have shown that MPD pathway could also be implicated in mannitol catabolism, which abrogates the existence of a mannitol cycle. And finally, analyses of double mutant revealed the existence of a new mannitol pathway which could parallel mannitol dehydrogenase pathway functions. During plant infection, mannitol metabolism could help pathogen to efficiently sequestrate plant hexoses. Gene expression studies revealed a regulated developmental control for mannitol pathway. Interconnections of mannitol metabolism with the central carbohydrate pathway suggested that it could regulate carbon metabolic fluxes. Mannitol constitutes then an easily mobilisable carbon store, for growth and dissemination but also to cope with stresses and to maintain hyphal turgor pressure.

\section{FUNDING}

Thierry Dulermo was supported by a doctoral scholarship from the Région Rhône-Alpes (Cluster 9), France.

\section{ACKNOWLEDGEMENTS}

We thank Anne-Marie Boisson for technical assistance and M. Wésolowski-Louvel for critical reading of the manuscript.

\section{REFERENCES}

1 Lewis, D.H., and Smith, D.C. (1967) Sugar alcohols (polyols) in fungi and green plants. Distribution, physiology and metabolism. New Phytol. 66, 143-184

2 Hult, K., Veide, A., and Gatenbeck, S. (1980) The distribution of the NADPH regenerating mannitol cycle among fungal species. Arch. Microbiol. 128, 253-255 
5463 Feofilova, E.P., Tereshina, V.M., Garibova, L.V., Zav'ialova, L.A., Memorskaia, A.S., 547 Maryshova, N.S. (2004) Germination of basidiospores of Agaricus bisporus. Prik1.

$548 \quad$ Biokhim. Mikrobiol. 40, 220-6

5494 Solomon, P.S., Waters, O.D., Jörgens, C.I., Lowe, R.G., Rechberger, J., Trengove, 550 R.D., and Oliver, R.P. (2006) Mannitol is required for asexual sporulation in the wheat 551

552

553

554

555

556

557

558

559

560

561

562

563

564

565

566

567

568

569

570

571

572

573

574

575

576

577

578

579

580

581

582

583

584

585

586

587

588

589

590

5 Ruijter, G.J., Bax, M., Patel, H., Flitter, S.J., van de Vondervoort, P.J., de Vries, R.P., vanKuyk, P.A., and Visser, J. (2003) Mannitol is required for stress tolerance in Aspergillus niger conidiospores. Eukaryot. Cell 2, 690-8

6 Martin, F., Ramstedt, M., Söderhäll, K., and Canet, D. (1988) Carbohydrate and amino acid metabolism in the ectomycorrhizal ascomycete Sphaerosporella brunnea during glucose utilization. A ${ }^{13} \mathrm{C}$ NMR study. Plant Physiol. 86, 935-940

7 Ceccaroli, P., Saltarelli, R., Cesari, P., Pierleoni, R., Sacconi, C., Vallorani, L., Rubini, P., Stocchi, V., and Martin, F. 2003. Carbohydrate and amino acid metabolism in Tuber borchii mycelium during glucose utilization: a (13)C NMR study. Fungal Genet. Biol. 39 , 168-75

8 Voegele, R.T., Hahn, M., Lohaus, G., Link, T., Heiser, I., and Mendgen, K. (2005) Possible roles for mannitol and mannitol dehydrogenase in the biotrophic plant pathogen Uromyces fabae. Plant Physiol. 137, 190-8

9 Jennings, D.B., Ehrenshaft, M., Pharr, D.M., and Williamson, J.D. (1998) Roles for mannitol and mannitol dehydrogenase in active oxygen-mediated plant defense. Proc. Natl. Acad. Sci. U S A. 95, 15129-15133

10 Vélëz, H., Glassbrook, N.J., and Daub, M.E. (2008). Mannitol biosynthesis is required for plant pathogenicity by Alternaria alternata. FEMS Microbiol. Lett. 285, 122-129

11 Jennings, D.B., Daub, M.E., Pharr, D.M., and Williamson, J.D. (2002) Constitutive expression of a celery mannitol dehydrogenase in tobacco enhances resistance to the mannitol-secreting fungal pathogen Alternaria alternata. Plant J. 32, 41-49

12 Hult, K., and Gatenbeck, S. (1978) Production of NADPH in the mannitol cycle and its relation to polyketide formation in Alternaria alternata. Eur. J. Biochem. 88, 607612

13 Solomon, P.S., Tan, K.C., and Oliver, R.P. (2005) Mannitol 1-phosphate metabolism is required for sporulation in planta of the wheat pathogen Stagonospora nodorum. Mol. Plant Mic. Int. 18, 110-5

14 Vélëz, H., Glassbrook, N.J., and Daub, M.E. (2007) Mannitol metabolism in the phytopathogenic fungus Alternaria alternata. Fungal. Genet. Biol. 4, 258-68

15 Dulermo, T., Rascle, C., Chinnici, G., Gout, E., Bligny, R., and Cotton, P. (2009) Dynamic carbon transfer during pathogenesis of sunflower by the necrotrophic fungus Botrytis cinerea: from plant hexoses to mannitol. New Phytol. 183, 1149-1162

16 Rolland, S., Jobic, C., Fèvre, M., and Bruel, C. (2003) Agrobacterium-mediated transformation of Botrytis cinerea, simple purification of monokaryotic transformants and rapid conidia-based identification of the transfer-DNA host genomic DNA flanking sequences. Curr. Genet. 44, 164-171

17 Malonek, S., Rojas, M.C., Hedden, P., Gaskin, P. and Tudzynski, B. (2004) The NADPH: cytochrome P450 reductase gene from Gibberella fujikuroi is essential for gibberellin biosynthesis. J. Biol. Chem. 279, 25075-25084 
59118 Hamada, W., Reignault, P., Bompeix, G., and Boccara, M. (1994) Transformation of

592

593

594

595

596

597

598

599

600

601

602

603

604

605

606

607

608

609

610

611

612

613

614

615

616

617

618

619

620

621

622

623

624

625

626

627

628

629

630

631

632

633

634

635

636

637

638

639

Botrytis cinerea with the hygromycin B resistance gene, hph. Curr. Genet. 26, 251-5

19 Church, G.M., Gilbert, W. (1984). Genomic sequencing. Proc Natl Acad Sci USA 81, 1991-5

20 Verwoerd, B., Dekker, M.,and Hoekema, A. (1989) A small-scale procedure for the rapid isolation of plant RNAs. Nucleic Acids Res. 17, 2362

21 Aubert, S., Gout, E., Bligny, R., Marty-Mazars, D., Barrieu, F., Alabouvette, J., Marty, F., and Douce, R. (1996) Ultrastructural and biochemical characterization of autophagy in higher plant cells subjected to carbon deprivation: control by the supply of mitochondria with respiratory substrates. J. Cell. Biol. 133, 1251-1263

22 Suvarna, K., Bartiss, A., and Wong, B. (2000) Mannitol-1-phosphate dehydrogenase from Cryptococcus neoformans is a zinc-containing longchain alcohol/polyol dehydrogenase. Microbiology 146, 2705-2713

23 Persson, B., Krook, M., andJornvall, H. (1991) Characteristics of short-chain alcohol dehydrogenases and related enzymes. Eur J Biochem 200, 537-543

24 Jörnvall, H., Persson, B., Krook, M., Atrian, S., Gonzàlez-Duarte, R., Jeffery, J., and Ghosh, D. (1995) Short-chain dehydrogenases/reductases (SDR). Biochem. 34, 600313

25 Solomon, P.S., Waters, O.D., and Oliver, R.P. (2007) Decoding the mannitol enigma in filamentous fungi. Trends Microbiol. 15, 257-262

26 Doehlemann, G., Berndt, P., and Hahn, M. (2006) Trehalose metabolism is important for heat stress tolerance and spore germination of Botrytis cinerea. Microbiology 152, 2625-2634

27 Jennings, D.H. (1984) Polyol metabolism in fungi. Adv. Microb. Physiol. 25, 149-193

28 Jobic, C., Boisson, A.M., Gout, E., Rascle, C., Fèvre, M., Cotton, P., and Bligny, R. (2007) Metabolic processes and carbon nutrient exchanges between host and pathogen sustain the disease development during sunflower infection by Sclerotinia sclerotiorum. Planta 226, 251-265

29 Olz, R., Larsson, K., Adler, L., and Gustafson, L. (1993) Energy flux and osmoregulation of Saccharomyces cerevisiae grown in chemostats under $\mathrm{NaCl}$ stress. J. Bacteriol. 175, 2205-2213

30 d'Enfert, C., and Fontaine, T. (1997) Molecular characterization of the Aspergillus nidulans tre $A$ gene encoding an acid trehalase required for growth on trehalose. Mol. Microbiol. 24, 203-216

31 Dixon, K.P., Xu, J.R., Smirnoff, N., and Talbot, N.J. (1999) Independent signaling pathways regulate cellular turgor during hyperosmotic stress and appressoriummediated plant infection by Magnaporthe grisea. Plant Cell 11, 2045-58

32 Clark, A.J., Blissett, K.J., and Oliver, R.P. (2003) Investigating the role of polyols in Cladosporium fulvum during growth under hyper-osmotic stress and in planta. Planta 216, 614-9

33 Lowe, R.G., Lord, M., Rybak, K., Trengove, R.D., Oliver, R.P., and Solomon, P.S. (2008) A metabolomic approach to dissecting osmotic stress in the wheat pathogen Stagonospora nodorum. Fungal Genet. Biol. 45, 1479-86

34 Liu, W., Leroux, P., and Fillinger, S. (2008) The HOG1-like MAP kinase Sak1 of Botrytis cinerea is negatively regulated by the upstream histidine kinase Bos1 and is not involved in dicarboximide- and phenylpyrrole-resistance. Fungal Genet. Biol. 45, 1062-1074

35 Ceccaroli, P., Saltarelli, R., Guescini, M., Polidori, E., Buffalini, M., Menotta, M., Pierleoni, R., Barbieri, E., and Stocchi, V. (2007) Identification and characterization of 
642

643

644

645

646

647

648

Tables :

\begin{tabular}{|c|c|c|c|c|c|c|}
\hline \multirow{3}{*}{$\begin{array}{l}\text { Allocation of } \\
\text { labelled carbon }\end{array}$} & \multicolumn{6}{|c|}{ Labelling transfer rate $\mu$ moles $\mathrm{g}^{-1} \mathrm{FW} \mathrm{h}^{-1}$} \\
\hline & \multicolumn{3}{|c|}{$3 \mathrm{mM}$ Glucose } & \multicolumn{3}{|c|}{$3 \mathrm{mM}$ Fructose } \\
\hline & WT & $\Delta B c m p d$ & $\Delta B c m t d h$ & WT & $\Delta B c m p d$ & $\triangle B c m t d h$ \\
\hline Glycogen & 0.9 & 1.4 & 0.3 & n.d. & n.d. & n.d. \\
\hline Mannitol C1/6 & 0.55 & 0.09 & 0.5 & n.d. & n.d. & n.d. \\
\hline Mannitol C2/5 & n.d. & n.d. & n.d. & 1.4 & 1.37 & 1 \\
\hline Trehalose C6 & 0.39 & 0.73 & 0.08 & n.d. & n.d. & n.d. \\
\hline
\end{tabular}

649

650

651

652

653

654

655

656

657

658
Table 1 Transfer rates of labelled hexoses through carbon mannitol metabolism in B. cinerea WT strain and single mutants.

Transfer rates were obtained from proton-decoupled ${ }^{13} \mathrm{C}-\mathrm{NMR}$ in vivo spectra of sunflower cotyledons infected by $B$. cinerea $(48 \mathrm{hpi})$. At time zero, $3 \mathrm{mM}\left[1-{ }^{13} \mathrm{C}\right]$ glucose or $3 \mathrm{mM}[2-$ $\left.{ }^{13} \mathrm{C}\right]$ fructose were added to perfusion medium. Sugar conversion was analysed during $3.5 \mathrm{~h}$. Transfer rates were determined at the end of the analysis period. Spectral conditions were as given in experimental procedures. Two independent experiments were done from separate infection series and cultures. A representative result is presented. n.d., not detected. 
662

\begin{tabular}{|c|c|c|c|c|c|c|c|c|}
\hline & \multicolumn{4}{|c|}{$2 \%$ Glucose } & \multicolumn{4}{|c|}{ 2\% Fructose } \\
\hline & WT & $\Delta B c m p d$ & $\Delta B c m t d h$ & $\begin{array}{l}\Delta B c m t d h \\
\Delta B c m p d\end{array}$ & WT & $\triangle B c m p d$ & $\Delta B c m t d h$ & $\begin{array}{l}\triangle B c m t d h \\
\triangle B c m p d\end{array}$ \\
\hline Glucose & 0.8 & 0.7 & 0.9 & 1.0 & n.d. & n.d. & n.d. & n.d. \\
\hline Fructose & n.d. & n.d. & n.d. & n.d. & 1.0 & 0.8 & & 0.4 \\
\hline Mannitol & 10.9 & 1.3 & 4.9 & 2.8 & 18.2 & 10.2 & 5.8 & 11.0 \\
\hline Tréhalose & 1.0 & 6.6 & n.d. & 6.4 & 0.3 & n.d. & n.d. & n.d. \\
\hline Arabitol & 0.3 & 0.6 & 0.7 & 0.5 & 1.0 & 1.5 & 1.3 & 1.3 \\
\hline Glycérol & 7.7 & 6.7 & 3.7 & 5.9 & 4.1 & 1.2 & 0.5 & 0.8 \\
\hline
\end{tabular}

Table 2 Impact of carbon sources on polyol and sugar content of WT, $\triangle B c m p d$, $\Delta B c m t d h, \Delta B c m p d \Delta B c m t d h$

$671 \Delta B c m p d \triangle B c m t d h$ strains were grown 2 -d on $2 \%$ glucose or $2 \%$ fructose. Sugars and polyols 672 were extracted and analysed by NMR in vitro spectroscopy, as described in experimental 673 procedures. Two independent experiments were done from separate cultures. A representative 674 result is presented. n.d., not detected

675

676

677 
678

679

680

681

682

683

684

685

686

687

688

689

690

691

692

693

694

695

696

697

698

699

700

701

702

703

704

705

706

707

708

709

710

711

712

713

714

715

716

717

718

719

720

721

722

723

724

725

726

727

\section{Figure Legends}

Figure 1 Model showing mannitol metabolism and main carbohydrate conversion pathways in fungi

Dotted arrows indicate series of enzymatic reactions.

Figure 2 Effect of mannitol pathway alteration on Bcmpd and Bcmtdh transcript levels, proteins and enzymatic activities

Mycelia were cultivated 2-d, on synthetic medium supplemented with $2 \%$ glucose. (A) Bcmpd and Bcmtdh gene expression. Bcmpd and Bcmtdh gene expression was measured by quantitative PCR using gene-specific primers and calibrated to BcactA transcripts. Amplifications were done in triplicate, from 3 independent biological replicates. Bars represent SD. (B) BcMPD and BcMTDH detection by western blot analysis. Gels were loaded with $75 \mu \mathrm{g}$ of proteins in each lane. Western blots were repeated twice, representative results are presented here. (C) BcMPD and BcMTDH enzymatic activities. Total mannitol-1phosphate dehydrogenase and mannitol dehydrogenase activities were measured from $50 \mu \mathrm{g}$ of proteins by monitoring absorbances changes of $\mathrm{NAD}(\mathrm{P})^{+/} \mathrm{NAD}(\mathrm{P}) \mathrm{H}$ at $340 \mathrm{~nm}$. Analyses were performed in duplicate from two independent biological replicates. A representative result is presented here.

Figure 3 Sugars content and Bcmpd and Bcmtdh transcript levels during in vitro development

(A) Sugars and polyols were extracted from 12-d-old fresh spores (conidia), germinating conidia (2, 4, and $6 \mathrm{~h}$ after activation of spore germination in rich liquid medium), young mycelium (2-d-old) and sporulating mycelium (6-d-old), after growth of WT strain, $\triangle B c m p d$ and $\triangle B c m t d h$ in the presence of glucose. Fungal extracts were then analysed by TLC as described in experimental procedures. Sugars and polyols were extracted from at least three independent biological replicates. A representative chromatography is presented here. (B) Total RNA was extracted from WT strain. Bcmpd and Bcmtdh gene expression was measured by quantitative PCR using gene-specific primers and calibrated to BcactA transcripts. RNA was extracted from at least three independent biological replicates. Bars represent SD. (C) Detection of BcMPD and BcMTDH in WT conidia and germinating conidia by Western blot analysis. Gels were loaded with $75 \mu \mathrm{g}$ of proteins in each lane. Proteins were extracted from at least three independent biological replicates. A representative western blot is presented here.

Figure 4 Intracellular sugar content and Bcmpd and Bcmtdh transcript levels in WT, $\triangle B c m p d$, and $\triangle B c m t d h$ during an osmotic stress response

Sugar, polyols and total RNA were extracted from young mycelium cultivated for $2 \mathrm{~d}$ on synthetic medium supplemented with $2 \%$ glucose and then transferred $0,0.5,1,4,6,9$ or $24 \mathrm{~h}$ on the same medium supplemented with $1 \mathrm{M} \mathrm{NaCl}$. (A) Analysis of mannitol, trehalose and glycerol content in WT and mannitol mutant strains was performed by TLC experiments. Culture transfers and TLC experiments were repeated three times from independent biological replicates. Representative chromatographies are presented here. (B) Bcmpd and Bcmtdh gene expression was quantified, in WT and mutant strains, by PCR using gene-specific primers and 
was calibrated to BcactA transcripts. Amplifications were done in triplicate from 3 independent biological replicates. Bars represent SD. (C) Western blot detection of BcMPD and BcMTDH in WT, $\Delta B c m p d$ and $\Delta B c m t d h$ strains. Gels were loaded with $75 \mu \mathrm{g}$ of proteins in each lane. Proteins were extracted from at least three independent biological replicates. Representative results are presented here.

Figure 5 Bcmpd deletion mutant reveals a mannitol phosphorylation pathway in B. cinerea

Proton-decoupled ${ }^{31} \mathrm{P}-\mathrm{NMR}$ spectra of PCA extracts of $\triangle B c m p d$ mycelium during growth in the presence of mannitol as sole carbon source. Extracts were prepared from 5 to $10 \mathrm{~g}$ of mycelium grown on $2 \%$ glucose $(\mathbf{A})$ or on $2 \%$ mannitol $(\mathbf{B})$. Peaks assignments are as follows: Mnt-1-P, mannitol-1-phosphate; Glcn-6-P, 6-phosphogluconate; $\alpha / \beta-G l c-6-\mathrm{P}, \alpha / \beta-$ glucose-6-phosphate; Tre-6-P, trehalose-6-phosphate; Gly-3-P, glycerol-3-phosphate; PGA, Phosphoglyceric acid; n.d., not determined. This experiment was done twice. Representative spectra are presented here.

Figure 6 Sugar and polyol content during in vitro development of $\Delta B c m p d \Delta B c m t d h$ Sugars and polyols were extracted from 2-day-old double mutant mycelium after growth in the presence of $2 \%$ glucose and analysed by TLC as described in experimental procedures. This experiment was done in triplicate using independent biological replicates. A representative chromatograph is presented here. 
851

852

853

854

855

856

857

858

859

860

861

862

863

864

865

866

867

868

869

870

871

872

873

874

875

876

877

878

879

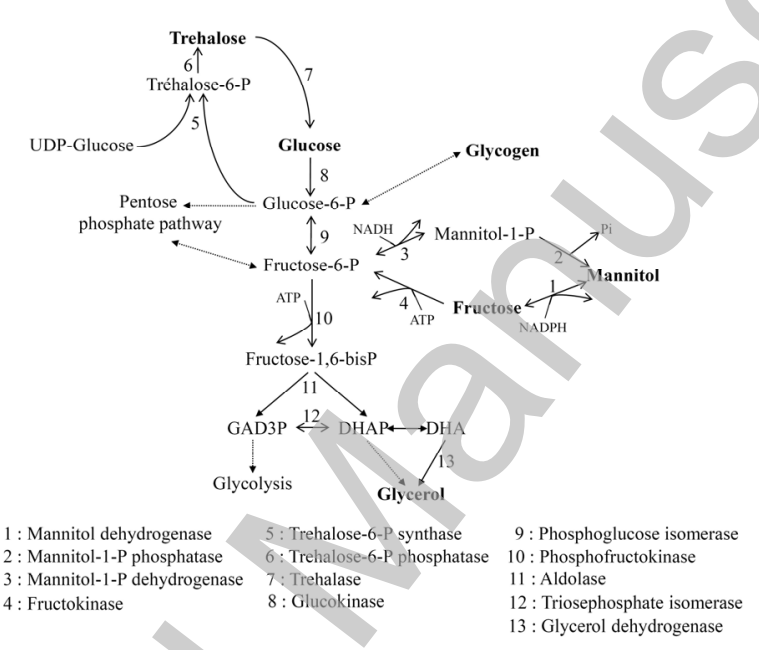

Figure1.tif

Licenced copy. Copying is not permitted, except with prior permission and as allowed by law. 
880

881

882

883

884

885

886

887

888

889

890

891

892

893

894

895

896

897

898

899

900

901

902

903

904

905

906

907
A

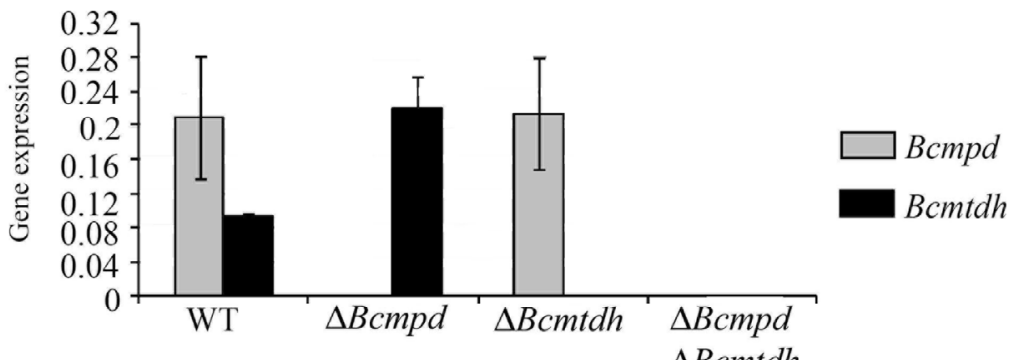

B

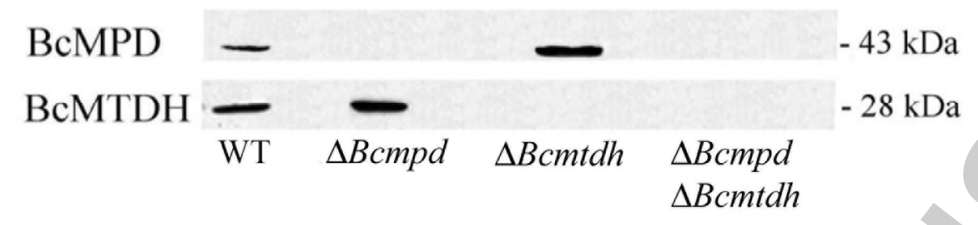

C

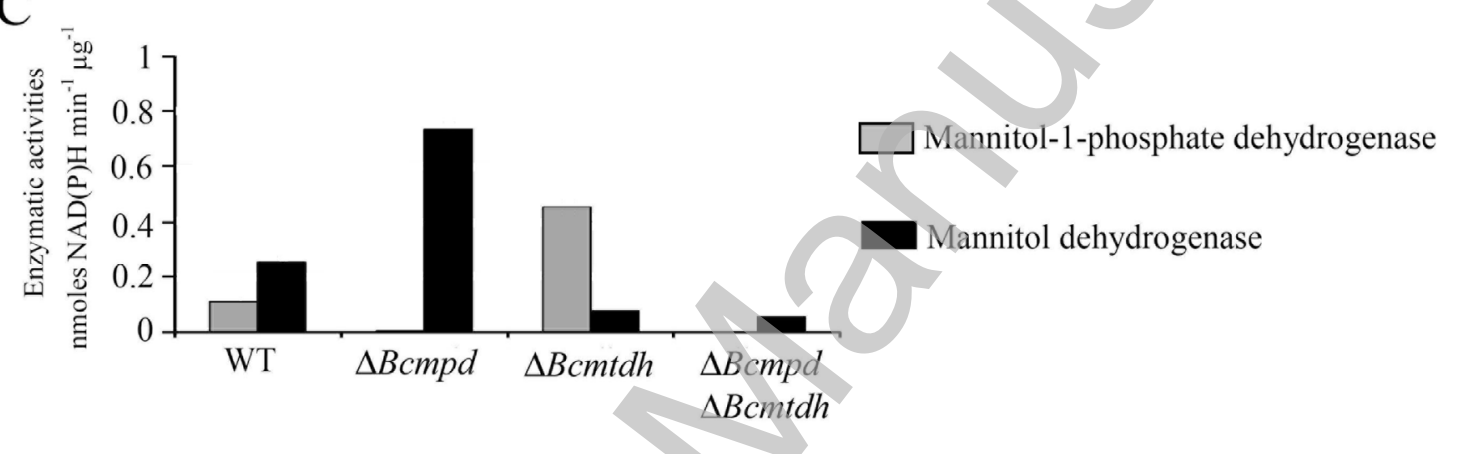

Figure2.tif 
908

909

910

911

912

913

914

915

916

917

918

919

920

921

922

923

924

925

926

927

928

929

930

931

932

933

934

935

936

937

938

939

940

941

942

943

944

945

946

947

948

949
A
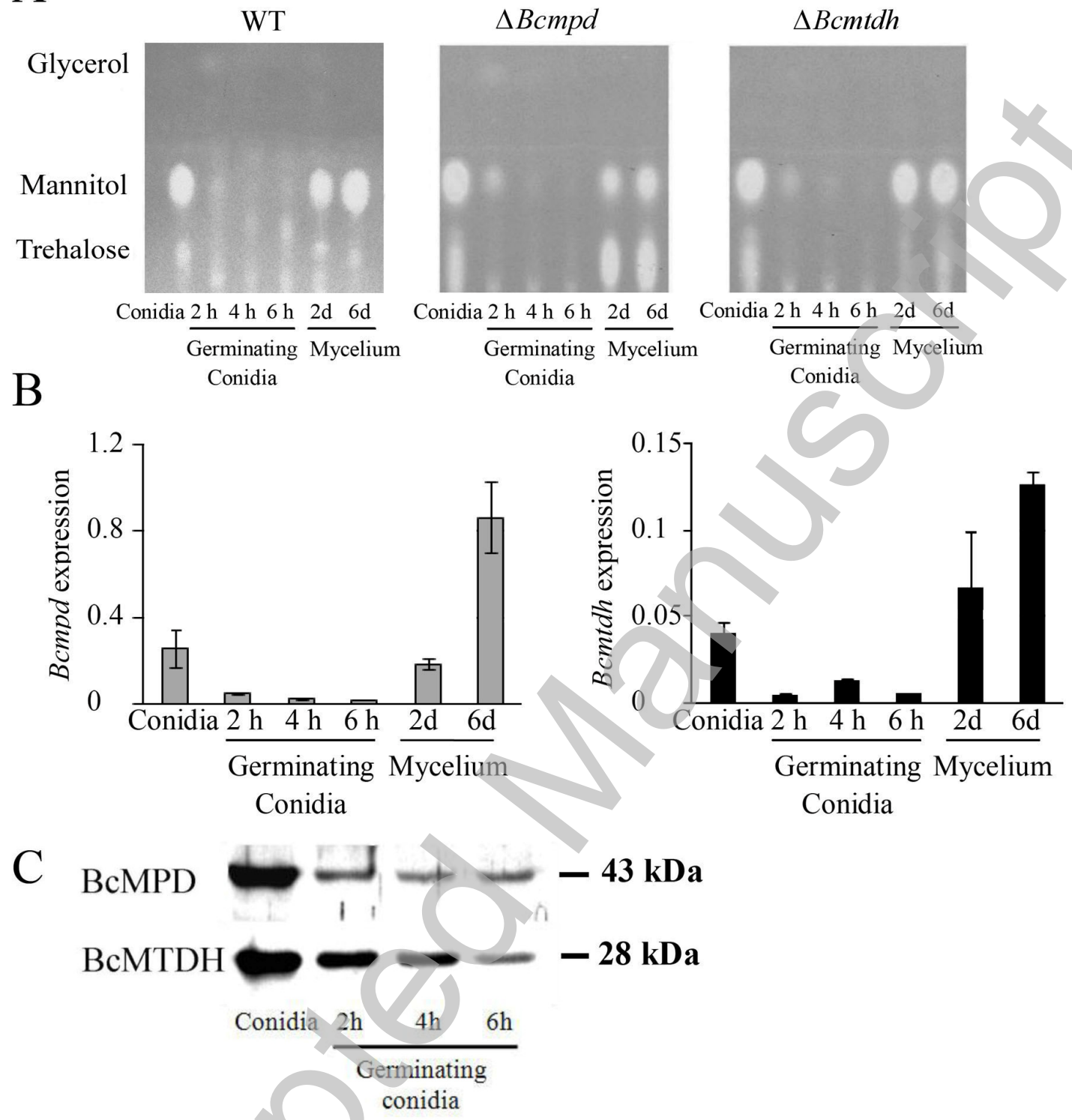

Figure3.tif 
953

954

955

956

957

958

959

960

961

A

WT

$\triangle B c m p d$
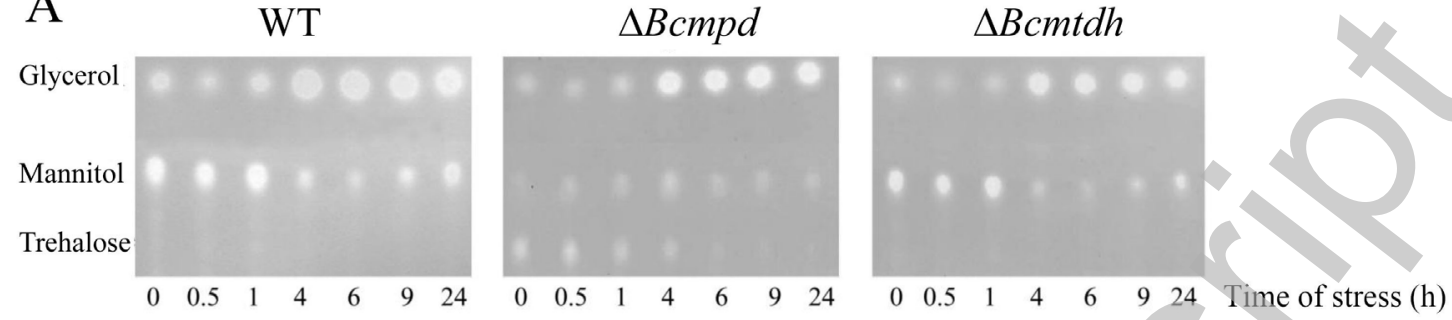

962

963

964

965

966

967

968

969

970

971

972

973

B
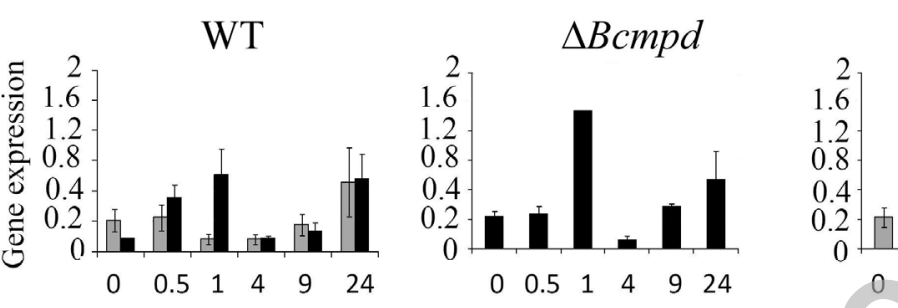

$\Delta B c m t d h$

C

WT

$\Delta B c m p d$

$\Delta B c m t d h$
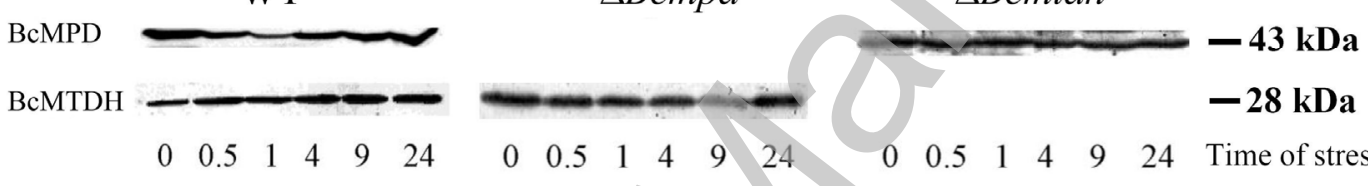

$-28 \mathrm{kDa}$

974

975

976

977

978

Figure4.tif 
979

980

981

982

983

984

985

986

987

988

989

990

991

992

993

994

995

996

997

998

999

1000

1001

1002

1003

1004

1005

1006

1007

1008

1009

1010

1011

1012

1013

1014

1015
A

$2 \%$ glucose

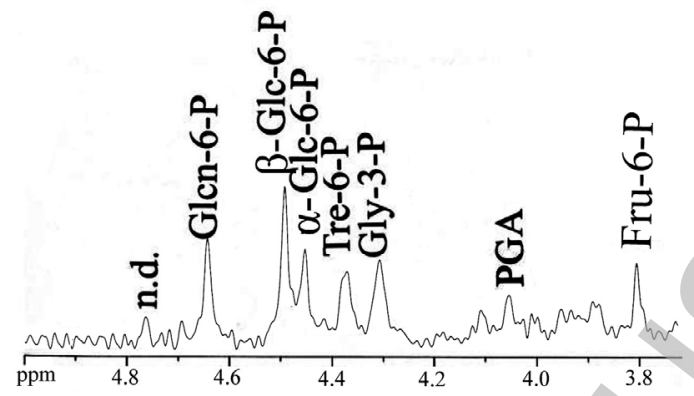

B

$2 \%$ mannitol

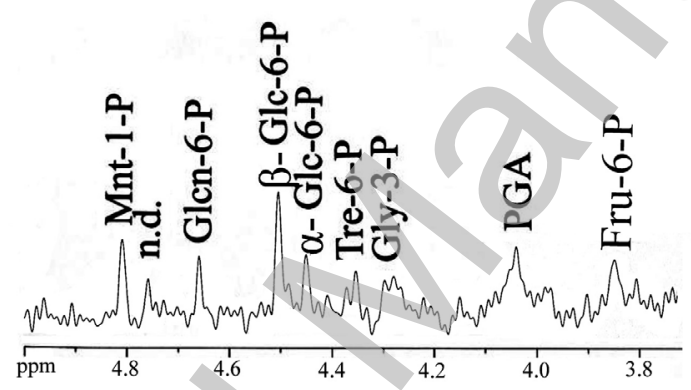

Figure5.tif 
1016

1017

1018

1019

1020

1021

1022

1023

1024

1025

1026

1027

1028

1029

1030

1031

1032

1033

1034

1035

1036

1037

1038

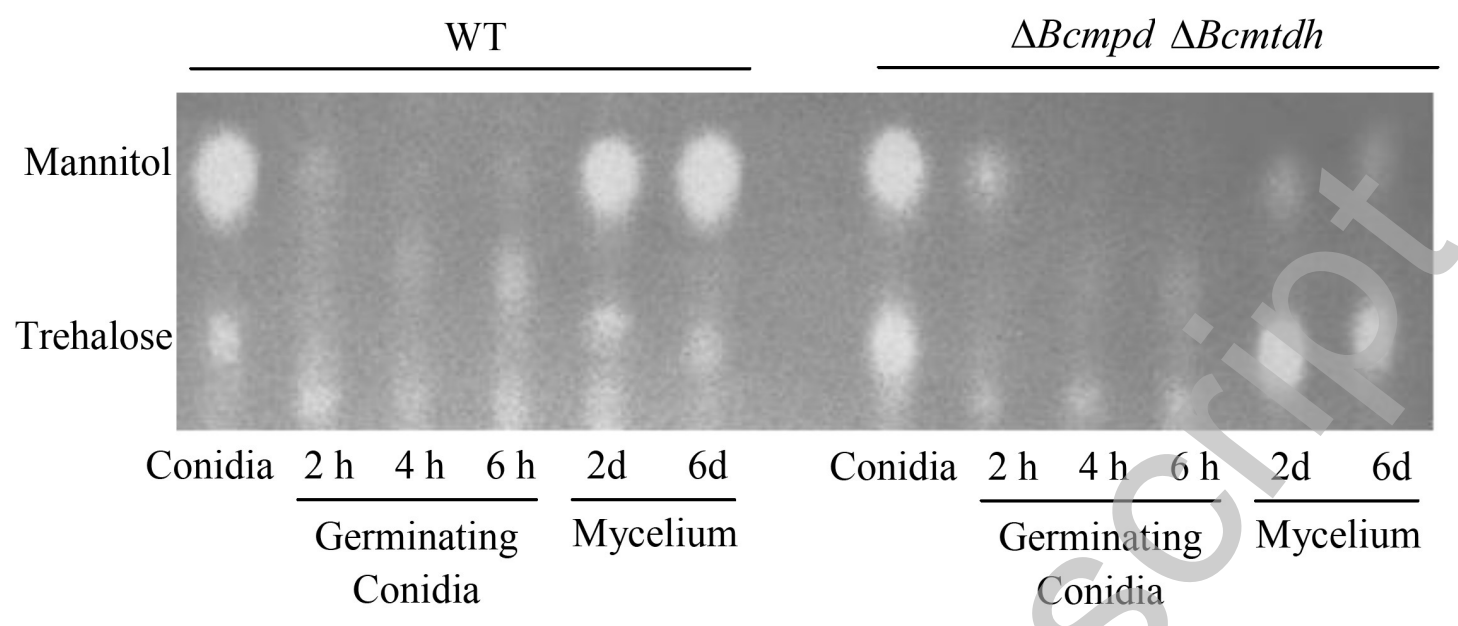

Figure6.tif

Licenced copy. Copying is not permitted, except with prior permission and as allowed by law. 